\title{
Ovarialkarzinom: Bevacizumab-Therapie bei Aszites effektiver
}

In der Primärtherapie des epithelialen Ovarialkarzinoms ab FIGO-Stadium IIIB ist die Therapie mit Bevacizumab und Paclitaxel/Carboplatin mittlerweile Standard. Eine neue Auswertung der GOG 0218-Studie lässt vermuten, dass davon vor allem Frauen mit tumorbedingtem Aszites profitieren.

D as Team um James S. Ferriss betrachtete Daten der BevacizumabZulassungsstudie GOG-0218 erneut [Ferriss JS et al. Gynecol Oncol. 2015; 139(1):17-22]. Sie schlossen Daten von 1.107 Patientinnen mit fortgeschrittenem Ovarialkarzinom ab FIGO-Stadium IIIB ein (Arm 1 und 3 der GOG0218-Studie): Frauen aus Arm 1 erhielten eine alleinige Standardchemotherapie aus 6 Zyklen Paclitaxel $\left(175 \mathrm{mg} / \mathrm{m}^{2}\right)$ plus Carboplatin (AUC 6); Frauen aus Arm 3 erhielten zusätzlich Bevacizumab (15 mg/kg q3w) sowie eine Erhaltungstherapie mit Bevacizumab über $10 \mathrm{Mo}$ nate. $80 \%$ der Patientinnen hatten einen Aszites $\left(>50,3 \mathrm{~cm}^{3}\right)$. Der Allgemeinzu- stand von Frauen mit Aszites war signifikant häufiger schlecht $(\mathrm{p}<0,001)$, und der Tumor war bei ihnen häufiger suboptimal mit einem Tumorrest $>1 \mathrm{~cm}$ reseziert worden $(\mathrm{p}=0,004)$.

Die Analyse bestätigte, dass das Auftreten von Aszites ein prognostischer Faktor für ein signifikant kürzeres Gesamtüberleben $(\mathrm{OS})$ ist $(\mathrm{p}<0,001)$. Insgesamt unterschied sich das OS in beiden Gruppen nicht signifikant, wie schon die ursprüngliche Datenanalyse gezeigt hatte. Allerdings lebten unter den Patientinnen mit Aszites die Frauen, die mit Standardchemotherapie plus Bevacizumab sowie anschließend mit Bevacizumab allein behandelt worden waren, signifikant länger progressionsfrei (PFS), im Vergleich zu Patientinnen unter alleiniger Chemotherapie (15,2 vs. 10,5 Monate). Außerdem war das OS unter Bevacizumab bei Patientinnen mit Aszites um 1 Monat länger (40,9 vs. 39,9 Monate) als bei den Frauen ohne Aszites.

Fazit: Diese Post-hoc-Analyse der Daten bestätigt Aszites als negativen prognostischen Faktor für das Gesamtüberleben bei Frauen mit fortgeschrittenem Ovarialkarzinom ab FIGO-Stadium IIIB. Erhalten die Betroffenen aber früh und kontinuierlich eine Standardchemotherapie und Bevacizumab, leben sie insgesamt signifikant länger und signifikant länger ohne Tumorprogress. Heike Grosse

Ferriss J et al. Ascites predicts treatment benefit of bevaciczumab in front-line therapy of advanced epithelial ovarian, fallopian tube and peritoneal cancers: An NRG Oncology/ GOG Study. Gynecol Oncol. 2015;139(1):17-22.

\section{Angiogenesehemmer verzögert Rezidiv beim Ovarialkarzinom}

\section{Die positiven Ergebnisse der OCEANS-Studie haben 2012 zur Zulassung von Bevacizumab zur Rezidivtherapie des Ovarialkarzinoms geführt. Ein signifi- kant längeres Gesamtüberleben zeigt auch die aktuelle Auswertung nicht.}

— ingeschlossen in die OCEANS-Stu_ die waren 484 Patientinnen mit platinsensiblem Ovarialkarzinom, die nach der Erstlinientherapie mit Carboplatin/Paclitaxel mindestens 6 Monate ohne Progress geblieben waren und Bevacizumab noch nicht in der Erstlinie erhalten hatten [Aghajanian $\mathrm{C}$ et al. J Clin Oncol. 2012;30(17):2039-45]. Sie bekamen Carboplatin/Gemcitabin über 6 Zyklen und randomisiert entweder Bevacizumab oder Placebo; letztere wurden dann als Monotherapie bis zur Progression weitergegeben. Das progressionsfreie Überleben (PFS) war für diese Patientinnen unter Bevacizumab um 4 Monate länger als in der Kontrollgruppe.

Die neuen Daten widmen sich den sekundären Endpunkten Sicherheit und Gesamtüberleben. Hier zeigten sich auch in der aktuellen Analyse der Studi- endaten keine signifikanten Unterschiede zwischen beiden Gruppen (33,6 vs. 32,9 Monate; Hazard Ratio 0,95).

Die detaillierte Aufschlüsselung in Sachen Sicherheit ergab, dass alle teilnehmenden Patientinnen mindestens 1 unerwünschtes Ereignis erlebten. Die Frauen in der Bevacizumab-Gruppe brachen die Studie aber wegen dieser Ereignisse häufiger ab als die Betroffenen der Kontrollgruppe (22,3 vs. 4,7\%). Wie von den Wissenschaftlern vorher postuliert, litten die Patientinnen der BevacizumabGruppe wesentlich häufiger unter Bluthochdruck (43,7 vs. 8,6\%), Proteinurie (21,5 vs. $4,3 \%)$ und Blutungen, die nicht das ZNS betrafen (68,0 vs. $32,6 \%)$. $1 \mathrm{~Pa}$ tientin in jeder Gruppe erlitt ein besonders schwerwiegendes Ereignis vom Grad 5: Unter Bevacizumab eine intrakranielle Blutung, unter Placebo einen
Myokard-Infarkt. Die jetzt vorliegenden Ergebnisse zeigen aber keine Hinweise auf neue Unverträglichkeitsmerkmale, die nicht schon 2012 veröffentlicht worden waren.

Auch wenn die aktuellen Daten in Bezug auf den sekundären Endpunkt Gesamtüberleben keinen signifikanten Unterschied zwischen den beiden Gruppen zeigen, zeichnet sich immerhin ein positiver Trend in der Bevacizumab-Gruppe hinsichtlich eines längeren progressionsfreien Überlebens ab.

Fazit: Obwohl die Patientinnen in der Bevacizumab-Gruppe insgesamt nicht signifikant länger überleben, tritt bei der Mehrzahl der Betroffenen ein Rezidiv erst später auf. Es wird also auch erst später eine weitere Therapie nötig. Heike Grosse

Aghajanian $C$ et al. Final overall survival and safety analyses of OCEANS, a phase 3 trial of chemotherapy with or without bevacizumab in patients with platin-sensitive recurrent cancer. Gynecol Oncol. 2015;139(1):10-6. 